\title{
Oxidative stress in the external intercostal muscles of patients with obstructive sleep apnoea
}

\author{
Esther Barreiro, Adam Nowinski, Joaquim Gea, Pawel Sliwinski
}

Thorax 2007;62:1095-1101. doi: 10.1136/thx.2006.069963

See end of article for authors' affiliations

Correspondence to: Dr Esther Barreiro, Muscle and Respiratory System Research Unit, IMIMHospital del Mar, Centro de Investigación en Red de Enfermedades Respiratorias (CibeRes), Experimental Sciences and Health Department (CEXS),

Universitat Pompeu Fabra, PRBB, C/Dr Aiguader 88, E-08003 Barcelona, Spain; ebarreiro@imim.es

Received 5 August 2006 Accepted 29 April 2007 Published Online First 15 June 2007

\begin{abstract}
Background: The external intercostal muscles are chronically exposed to increased inspiratory loading and to continuous hypoxia-reoxygenation cycles in patients with obstructive sleep apnoea syndrome (OSAS). It was therefore hypothesised that oxidative stress levels would be increased in these muscles, and that treatment with continuous positive airway pressure (CPAP) would modify the oxidative stress levels and improve muscle dysfunction.

Methods: A case-control study and a case-case study were conducted on the external intercostal muscles of 12 patients with severe OSAS (before and after 6 months of treatment with CPAP) and 6 control subjects. Reactive carbonyl groups, malondialdehyde (MDA)-protein and hydroxynonenal (HNE)-protein adducts, antioxidant enzyme levels, 3-nitrotyrosine and fibre type proportions were measured using immunoblotting and immunohistochemistry.

Results: Compared with controls, the intercostal muscles of patients with OSAS had higher levels of protein carbonylation (median values 3.06 and 2.45, respectively, $p=0.042$ ), nitration (median values 1.64 and 1.05 , respectively, $p=0.019$ ) and proportions of type I fibres (median values $57 \%$ and $48 \%$, respectively, $p=0.035$ ) and reduced respiratory muscle endurance (median values 3.2 and $9.5 \mathrm{~min}$, respectively, $p=0.001)$. Positive correlations were found between MDA-protein and HNE-protein adducts $(r=0.641$, $p=0.02$ and $r=0.594, p=0.05$, respectively) and 3-nitrotyrosine $(r=0.625, p=0.03)$ and the apnoeahypopnoea index (AHI) in all the patients with OSAS. Although treatment with CPAP significantly improved the $\mathrm{AHI}$ and oxygen desaturation, muscle oxidative stress levels and respiratory muscle endurance were not affected.

Conclusions: This study suggests that inspiratory muscle performance is not completely restored after longterm treatment with CPAP.
\end{abstract}

O bstructive sleep apnoea syndrome (OSAS) is a highly prevalent condition characterised by recurrent upper airway obstruction during sleep. ${ }^{1}$ As inspiratory muscles of patients with OSAS must generate greater negative intrathoracic pressures than usual in order to overcome the obstructive apnoea, they may be subjected to mechanical overloading. ${ }^{2}$ The diaphragm appears to be the primary inspiratory muscle during apnoea in some patients with OSAS, while the intercostals muscles are the main inspiratory muscles in other patients. ${ }^{2}$ Indeed, hypercapnic respiratory failure has been recognised in patients with severe OSAS. ${ }^{3}$ Furthermore, in patients with OSAS, daytime ventilatory muscle force and diaphragm strength were both shown to be preserved while inspiratory muscle endurance was significantly reduced. ${ }^{4}$ Interestingly, the administration of nocturnal continuous positive airway pressure (CPAP) to these patients attenuated the decrease in respiratory muscle resistance. ${ }^{4}$ However, the underlying mechanisms of this respiratory muscle dysfunction in OSAS are still unknown.

Reactive oxygen species (ROS) are usually synthesised at low levels in muscles and are required for normal force generation. Their levels progressively increase in response to muscle activation, and excessive production of ROS results in oxidative stress which, in turn, leads to a decline in muscle force production. Oxidative stress has been implicated in the pathogenesis of different chronic condition ${ }^{56}$ and in aging. ${ }^{7}$ Importantly, increased superoxide anion production and lipid peroxidation have been reported in the blood of patients with OSAS with and without cardiovascular disease. ${ }^{89}$ Furthermore, a growing body of evidence shows that oxidative stress is one of the mechanisms clearly involved in skeletal muscle dysfunction in different conditions such as chronic obstructive pulmonary disease (COPD) and sepsis. ${ }^{10-14}$

Few studies have examined whether oxidative stress develops in the respiratory muscles of patients with OSAS. On the grounds that the production of ROS within skeletal muscle fibres is regulated in part by strong muscle contractions, and that during night time patients with severe OSAS are chronically exposed to increased inspiratory muscle loading and to continuous hypoxia-reoxygenation cycles, we hypothesised that their respiratory muscles might generate increased levels of oxidative stress. To test this hypothesis we first sought to determine the levels of protein carbonylation and nitration in the external intercostal muscles of patients with OSAS compared with a control group of subjects and to examine whether CPAP treatment can reduce these levels. We then explored the levels of several antioxidant mechanisms in these muscles and finally investigated whether oxidative stress indices were associated with either the severity of the disease or respiratory muscle function of such patients.

Abbreviations: $\mathrm{AHI}$, apnoea-hypopnoea index; COPD, chronic obstructive pulmonary disease; CPAP, continuous positive airway pressure; DNP, 2-4 dinitrophenylhydrazone; ESS, Epworth sleepiness scale; GAPDH, glyceraldehyde-3-phosphate dehydrogenase; $\mathrm{HNE}$, hydroxynonenal; HRP, horseradish peroxidase; MDA, malondialdehyde; $O D$, optical density; OSAS, obstructive sleep apnoea syndrome; PVDF, polyvinylidene difluoride; $\mathrm{ROS}$, reactive oxygen species; $\mathrm{SaO}_{2}$, arterial oxygen saturation; SOD, superoxide dismutase 


\section{METHODS}

\section{Subjects}

All individuals were Caucasian. Twelve consecutive male patients with a polysomnographically confirmed diagnosis of OSAS (clinic referrals recently diagnosed and without CPAP therapy) were included. Six male healthy non-smoker controls with similar anthropometric characteristics without OSAS, as indicated by clinical history and a negative sleep study (polysomnography), were also recruited from the general population. The sample size of both patient and control populations was calculated on the basis of formerly published studies by our group where similar physiological and biological approaches were used. ${ }^{10} 1213$ Exclusion criteria included previous treatment with CPAP, female sex in order to avoid sexrelated effects on muscles, ${ }^{15}$ COPD, bronchial asthma, cardiovascular disease, chronic metabolic diseases including diabetes, suspected paraneoplastic or myopathic syndromes and/or treatment with drugs known to alter muscle structure and/or function (see additional information in online supplement available at http://thorax.bmj.com/supplemental).

\section{Study design}

This study had a twofold design: case-control (OSAS patients vs controls) and case-case (OSAS patients before and after treatment with CPAP). The study was designed in accordance with both the ethical standards on human experimentation in our institution and the World Medical Association guidelines for research on human beings. The Ethics Committee on Human Investigation at the Institute of Tuberculosis and Lung Diseases in Warsaw approved all experiments. Informed written consent was obtained from all individuals.

\section{Clinical, nutritional and functional assessment}

Clinical evaluation included medical history, a complete physical examination, thorax radiography and electrocardiogram. Nutritional evaluation included anthropometric and analytical parameters. Pulmonary and respiratory muscle function, general exercise capacity and peripheral muscle strength were evaluated ${ }^{16-20}$ (see additional information in online supplement available at http://thorax.bmj.com/supplemental).

\section{Polysomnography}

Standard polysomnographic examination was performed in the sleep laboratory according to the standards of the American Sleep Disorders Association. ${ }^{21}$ The diagnosis of OSAS was established by obtaining an apnoea-hypopnoea index (AHI) of $>10 /$ h of sleep. ${ }^{4921}$ When the diagnosis of OSAS was clearly established, patients underwent a second polysomnographic examination for manual CPAP titration. CPAP treatment (REMstar, Respironics, Murrysville, Pennsylvania, USA) was applied to all patients for at least 6 months. Adherence to CPAP treatment over this period was monitored by the built-in time counter in the CPAP device. Good compliance with treatment was established as a mean usage of CPAP of $>4 \mathrm{~h} /$ night. All patients also completed the Epworth Sleepiness Scale (ESS) ${ }^{22}$ (normal: ESS score <10; borderline: ESS score 10-12; excessive sleepiness: ESS score $>12$ ) before and after the 6-month CPAP treatment period. CPAP treatment was administered to all patients 7-10 days after undergoing baseline muscle biopsy surgery (see additional information in online supplement available at http://thorax.bmj.com/supplemental).

\section{Muscle biopsies}

Biopsy specimens $(\approx 40 \mathrm{mg})$ were taken from the external intercostal muscles before and after the 6-month period of treatment with CPAP following procedures published else- where $^{23}$ (see additional information in online supplement available at http://thorax.bmj.com/supplemental).

\section{Biological muscle studies}

All the muscle biology analyses were conducted in a blind manner in the same laboratory at Hospital del Mar-IMIM, Barcelona.

\section{Immunoblotting}

The effects of oxidants on muscle proteins and lipids were evaluated according to methods published elsewhere. ${ }^{10}{ }^{12-14}$ Immunoblotting experiments were specifically designed so that muscle homogenates from control subjects $(n=6)$ and from patients with OSAS before $(n=12)$ and after $(n=12)$ CPAP treatment were always run together and kept in the same order. The following antibodies were used to detect the different antigens and phenomena: anti-DNP moiety antibody (Oxyblot kit, Chemicon International Inc, Temecula, California, USA), anti-MDA antibody (Academy Bio-Medical Company Inc, Houston, Texas, USA), anti-HNE antibody (Alpha Diagnostics International Inc, San Antonio, Texas, USA), anti-3-nitrotyrosine antibody (Cayman Chemical Inc, Ann Arbor, Michigan, USA), anti-Mn SOD antibody (StressGen, Victoria, BC, Canada) and anti-catalase antibody (Calbiochem, San Diego, California, USA). Blots were scanned with an imaging densitometer and optical densities (OD) of specific proteins were quantified with Diversity Database 2.1.1 (BioRad, Philadelphia, Pennsylvania, USA) (see additional information in online supplement available at http://thorax.bmj.com/supplemental).

\section{Immunohistochemistry}

Immunohistochemical experiments were performed according to methods published elsewhere. ${ }^{10} 12$ Briefly, myosin heavy chain I and II isoforms were identified on $3 \mu \mathrm{m}$ muscle paraffin embedded sections using anti-myosin heavy chain I (clone MHC, Biogenesis Inc, Poole, UK) and anti-myosin heavy chain II (clone MY-32, Sigma, St Louis, Missouri, USA) primary antibodies, respectively, as well as markers of oxidative stress.

\section{Statistical analysis}

Data are presented as median (interquartile range) values in table 1 and as box and whisker plots in the figures. MannWhitney non-parametric tests were used for comparisons between controls and patients with OSAS at baseline, and the Wilcoxon test was used to compare variables before and after CPAP treatment in patients with OSAS. Spearman's coefficient was used to assess correlations between biological and physiological variables among the patients with OSAS. A p value of $\leqslant 0.05$ was considered significant.

\section{RESULTS}

\section{Characteristics of study subjects}

Table 1 shows the main characteristics of the control subjects and patients with OSAS at baseline and after CPAP treatment. No significant differences in age, nutritional status (as assessed by body mass index) or lung function parameters were observed between control subjects and patients with OSAS before CPAP treatment. However, the following sleep variables were significantly reduced in the patients before CPAP treatment compared with control subjects: mean arterial oxygen saturation $\left(\mathrm{SaO}_{2}\right)$ during the night, nadir $\mathrm{SaO}_{2}$ and time spent at $\mathrm{SaO}_{2}<90 \%$. The ESS score was significantly increased in patients $(>12)$, indicating excessive sleepiness compared with controls $(<10)$. Interestingly, all these parameters significantly improved after CPAP treatment for 6 months. Patients adequately complied with CPAP treatment, with a mean usage of $5.4 \mathrm{~h}$. Exercise capacity and respiratory 
Table 1 Main characteristics and functional variables of study subjects

\begin{tabular}{|c|c|c|c|}
\hline & $\begin{array}{l}\text { Controls } \\
(n=6)\end{array}$ & $\begin{array}{l}\text { Patients with OSAS } \\
\text { before treatment } \\
(n=12)\end{array}$ & $\begin{array}{l}\text { Patients with OSAS } \\
\text { after treatment } \\
(n=12)\end{array}$ \\
\hline Age (years) & $43.5(13)$ & $43(10)$ & $43(11)$ \\
\hline$B M I\left(\mathrm{~kg} / \mathrm{m}^{2}\right)$ & $33.9(6.2)$ & $35.9(8)$ & $34.3(6)$ \\
\hline AHI (events/h) & $4(1.5)$ & $78(41.2)^{\star \star \star}$ & $3(3.8)+t \dagger$ \\
\hline Mean $\mathrm{SaO}_{2}(\%)$ & $95(3.5)$ & $85(7.5)^{\star \star *}$ & $94(4)+\dagger \dagger$ \\
\hline Nadir $\mathrm{SaO}_{2}(\%)$ & $92(5)$ & $70(26.5)^{\star \star \star}$ & $88(5)+\dagger \dagger$ \\
\hline Time $\mathrm{SaO}_{2}<90 \%(\%)$ & $1(3.5)$ & $74(22.5)^{\star \star \star}$ & $1(15)+\dagger \dagger$ \\
\hline $\mathrm{CPAP}\left(\mathrm{cm} \mathrm{H}_{2} \mathrm{O}\right)$ & - & - & $9(3.5)$ \\
\hline CPAP time (h) & - & - & $5(1.2)$ \\
\hline ESS score & $5.5(5.2)$ & $16(5.5)^{\star * *}$ & $9.5(7.2)+\dagger \dagger$ \\
\hline $\mathrm{FEV}_{1}(\%$ pred) & $93(28)$ & $95(11)$ & 99 (12) \\
\hline FVC ( $\%$ pred) & $93(24)$ & $111(20)$ & $111(15)$ \\
\hline $\mathrm{FEV}_{1} / \mathrm{FVC}(\%)$ & $80(6)$ & $75(7)$ & $73(3)$ \\
\hline FRC (\% pred) & NA & 87 (19) & $96(20)$ \\
\hline TLCO (\% pred) & NA & $118(36)$ & $125(41)$ \\
\hline $\mathrm{PaO}_{2}(\mathrm{~mm} \mathrm{Hg})$ & $74(8)$ & $74(15)$ & $78(12)$ \\
\hline $\mathrm{PaCO}_{2}(\mathrm{~mm} \mathrm{Hg})$ & $40.1(5)$ & $41.5(3.1)$ & $39.6(2.9)$ \\
\hline MIP (\% pred) & N.A. & $91(21)$ & $108(33)$ \\
\hline Tlim (min) & $9.5(1.6)$ & $3.2(4.3)^{\star \star *}$ & $2.1(0.2)$ \\
\hline Handgrip, (\% pred) & NA & $115(69)$ & $131(72)$ \\
\hline 6-min walking distance (\% pred) & NA & $92(13)$ & 90 (19) \\
\hline Type I fibres (\%) & $48(11)$ & $57(15)^{*}$ & $41(27)+\dagger$ \\
\hline Type II fibres (\%) & $52(11)$ & $43(15)^{*}$ & 59 (27) t† \\
\hline Type I fibre area $\left(\mu \mathrm{m}^{2}\right)$ & $1813(1018)$ & $2206(585)$ & $1970(266)$ \\
\hline Type II fibre area $\left(\mu \mathrm{m}^{2}\right)$ & 2219 (1005) & $2206(1079)$ & $2463(633)$ \\
\hline \multicolumn{4}{|c|}{$\begin{array}{l}\text { Data are presented as median (interquartile range). } \\
\mathrm{BMI} \text { body mass index; } \mathrm{SaO}_{2} \text {, arterial oxygen saturation; } \mathrm{CPAP} \text {, continuous positive airway pressure; } \mathrm{ESS} \text {, Epworth } \\
\text { Sleepiness scale; } \mathrm{FEV}_{1} \text {, forced expiratory volume in } 1 \mathrm{~s} \text {; pred, predicted; } \mathrm{FVC} \text {, forced vital capacity; FRC, functional } \\
\text { residual capacity (as expressed by intrathoracic gas volume); TLCO, carbon monoxide transfer factor; MIP, maximal } \\
\text { inspiratory pressure, Tlim, endurance time. } \\
{ }^{*} \mathrm{p} \leqslant 0.05,{ }^{* \star} \mathrm{p} \leqslant 0.01,{ }^{* \star *} \mathrm{p} \leqslant 0.001 \text { control subjects vs OSAS patients before treatment. } \\
t \mathrm{t} \leqslant 0.05,+t p \leqslant 0.01,+\dagger+p \leqslant 0.001 \text { OSAS patients before vs after treatment. }\end{array}$} \\
\hline
\end{tabular}

and peripheral muscle strength were preserved in patients with OSAS and were not significantly modified after CPAP treatment. In contrast, endurance time of the respiratory muscles was significantly reduced in patients with OSAS compared with controls and did not improve after CPAP treatment.

\section{Muscle structure}

The proportion of type I fibres was significantly increased while the proportion of type II fibres was decreased in the intercostal muscles of patients with OSAS compared with controls (table 1). Interestingly, after CPAP treatment the proportions of fibres were significantly reversed to values observed in control subjects.

\section{Protein carbonylation}

\section{Total carbonyl group formation}

The intercostal muscles of the patients had significantly higher levels of protein carbonylation than control muscles (median values 3.06 and 2.45, respectively, $\mathrm{p}=0.042$; fig $1 \mathrm{~A}$ ). In the patients with OSAS a significant inverse relationship was found between protein carbonylation and nadir $\mathrm{SaO}_{2}(r=-0.711$, $\mathrm{p}=0.032)$. An almost significant inverse correlation $(r=-0.643, \mathrm{p}=0.1)$ was also found between protein carbonylation and the endurance time of the respiratory muscles in patients with OSAS. No other correlations were observed between protein carbonylation and lung or respiratory muscle function, sleep variables or exercise tolerance. The levels of total protein carbonylation were not significantly reduced after CPAP treatment in patients with OSAS (median value 3.39, $\mathrm{p}=0.859$; fig lA). Immunostaining with anti-DNP antibody revealed the presence of carbonyl groups diffusely localised within the intercostal muscle fibres in patients with OSAS and control subjects (fig 1B).

\section{MDA-protein adduct formation}

The intercostal muscles of the patients with OSAS had significantly higher levels of MDA-protein adducts than those of the controls (median values 1.91 and 1.24, respectively, $\mathrm{p}=0.005$; fig 2A). Among all patients with OSAS, the intensity of total intercostal MDA-protein adducts directly correlated with the severity of their disease as measured by the AHI (fig 2B). No other correlations were observed between MDAprotein adduct levels and lung or respiratory muscle function, sleep variables or exercise tolerance. The levels of MDA-protein adducts were not significantly reduced after CPAP treatment in patients with OSAS (median value 2.07, $\mathrm{p}=1.0$; fig $2 \mathrm{~A}$ ).

\section{HNE-protein adducts}

The intercostal muscles of the patients with OSAS had significantly higher levels of HNE-protein adducts than control muscles (median values 1.15 and 0.63 , respectively, $\mathrm{p}=0.002$; fig 3). Among all patients with OSAS, the intensity of total intercostal HNE-protein adducts directly correlated with the severity of their disease as measured by the AHI $(r=0.594$, $p=0.05)$. No other correlations were observed between HNEprotein adducts and lung or respiratory muscle function, sleep variables or exercise tolerance. The levels of HNE-protein adducts were not significantly reduced after CPAP treatment in patients with OSAS (median value $0.87, \mathrm{p}=0.327$; fig 3 ).

\section{Protein tyrosine nitration}

The intercostal muscles of the patients with OSAS had significantly greater levels of tyrosine nitrated proteins than control muscles (median values 1.64 and 1.05, respectively, $\mathrm{p}=0.019$; fig 4A). Among all patients with OSAS, the intensity of total 3-nitrotyrosine immunoreactivity positively correlated with the severity of their disease as measured by the AHI (fig 4B). No other correlations were observed between protein 


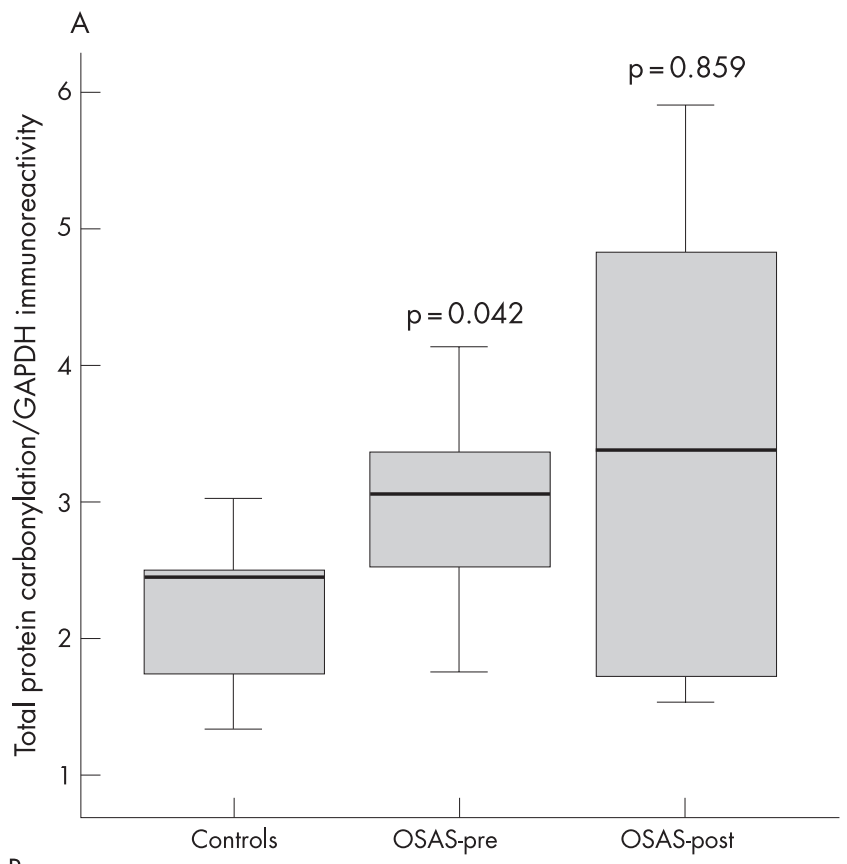

B
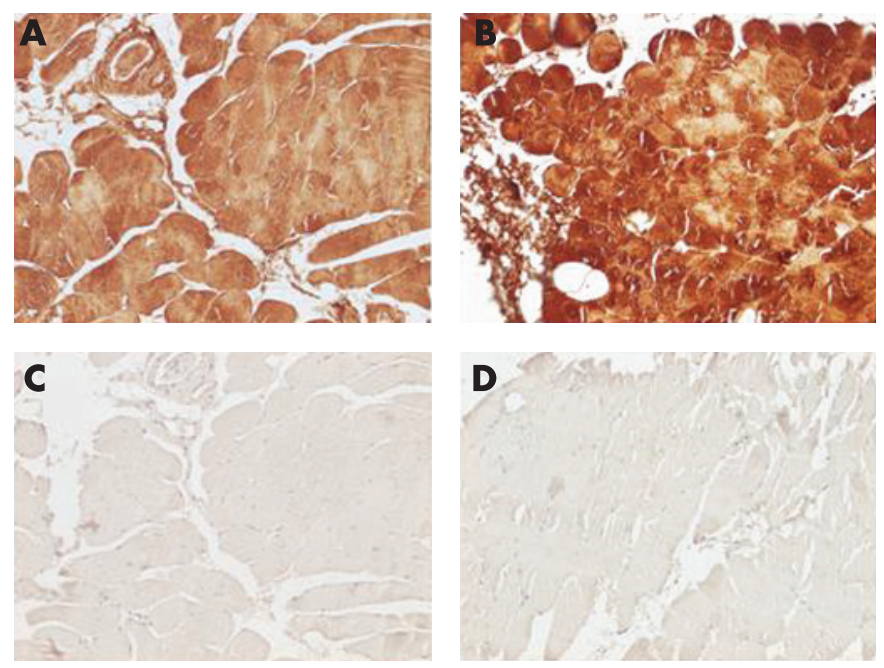

Figure 1 (A) Optical densities in the box plots are expressed as the ratio of the optical densities of total reactive carbonyl groups to those of glyceraldehyde-3-phosphate dehydrogenase (GAPDH). Standard box plots with median (25th and 75th percentiles) and whiskers (at minimum and maximum values) are depicted. At baseline, reactive carbonyl groups were significantly greater in the external intercostal muscles of patients with severe obstructive sleep apnoea syndrome (OSAS) than in the muscles of control subjects (median values 3.06 and 2.45 , respectively, $p=0.042$ ). Total muscle reactive carbonyls were not significantly modified after a 6 month period of treatment with continuous positive airway pressure (CPAP) (median value 3.39, $p=0.859$ ). OSAS-pre, patients with OSAS before treatment with CPAP; OSAS-post, patients with OSAS after 6 months of treatment with CPAP. (B) Immunohistochemical localisation of carbonylmodified proteins in the muscle fibres of the intercostal muscles of one control subject (panel A) and one patient with severe OSAS (panel B) (magnification 200 $\times$ ). Anti-DNP antibody detected positive staining diffusely localised within the fibres of the external intercostal muscles (panels A and B). Removal of primary anti-DNP antibody completely eliminated positive carbonyl-modified protein staining (panels $C$ and D). Note that the staining was more intense in the intercostal muscles of the patient than in the control muscle.

tyrosine nitration and lung or respiratory muscle function, sleep variables or exercise tolerance. The levels of 3-nitrotyrosine immunoreactivity were not significantly reduced after CPAP treatment in patients with OSAS (median value $1.27, \mathrm{p}=0.575$;
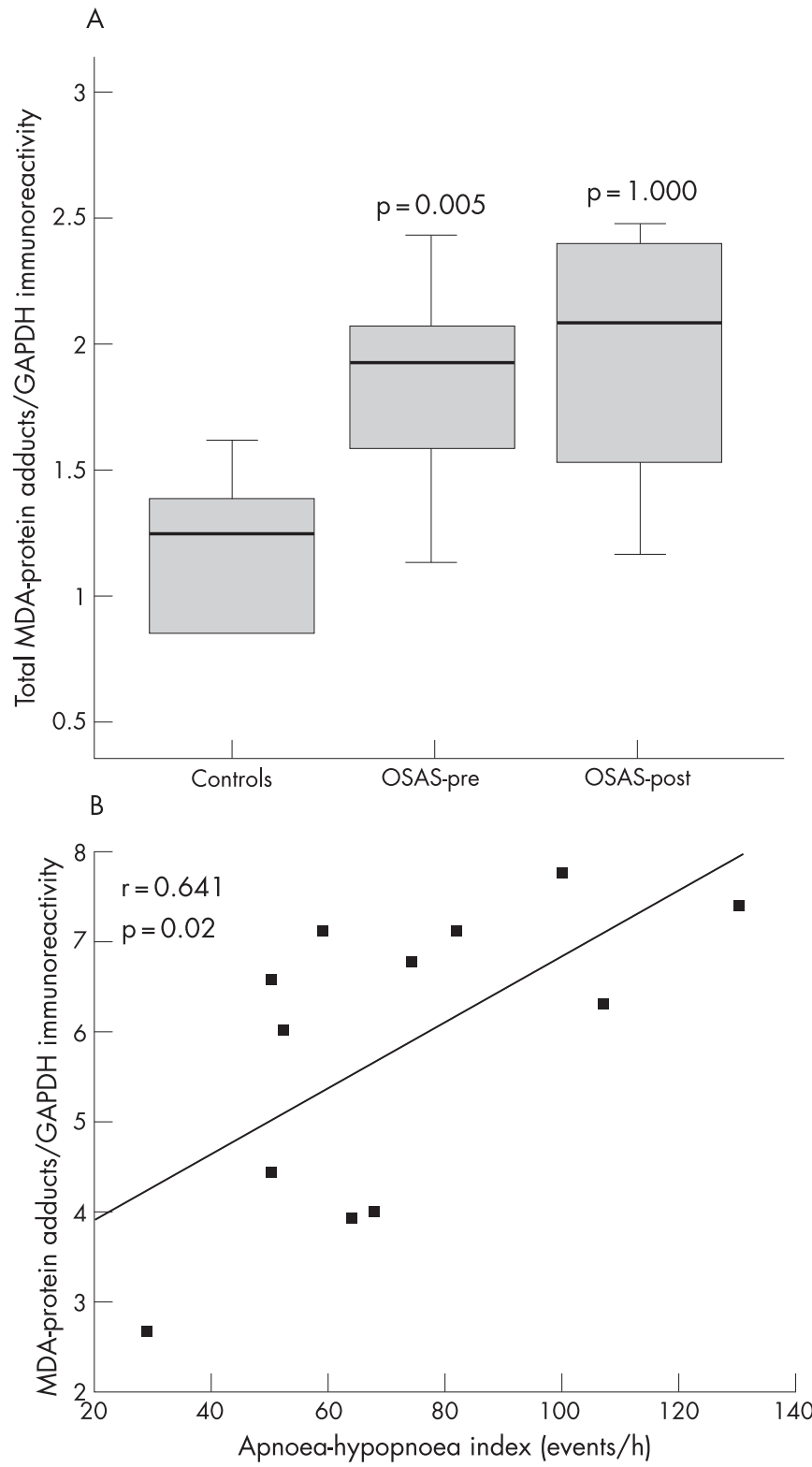

Figure 2 (A) Optical densities in the box plots are expressed as the ratio of the optical densities of total malondialdehyde (MDA)-protein adducts to those of glyceraldehyde-3-phosphate dehydrogenase (GAPDH). Standard box plots with median (25th and 75th percentiles) and whiskers (at minimum and maximum values) are shown. At baseline, total levels of MDA-protein adducts were significantly greater in the external intercostal muscles of patients with severe obstructive sleep apnoea syndrome (OSAS) than in control muscles (median values 1.91 and 1.24 , respectively, $\mathrm{p}=0.005$ ). Total muscle MDA-protein adducts were not significantly modified after a 6-month period of treatment with continuous positive airway pressure (CPAP) (median value 2.07, $p=1.0$ ). OSAS-pre, patients with OSAS before treatment with CPAP; OSAS-post, patients with OSAS after 6 months of treatment with CPAP. (B) Overall, in patients with OSAS the ratio of the optical densities of total MDA-protein adducts to those of GAPDH directly and significantly correlated with the apnoea-hypopnoea index (events/h).

fig 4A). Immunostaining with anti-nitrotyrosine antibody revealed the presence of tyrosine nitrated proteins diffusely localised within the intercostal muscle fibres in both patients with OSAS and controls (fig 4C).

\section{Antioxidant enzymes}

Both manganese superoxide dismutase and catalase were detected in the external intercostal muscles of patients with 


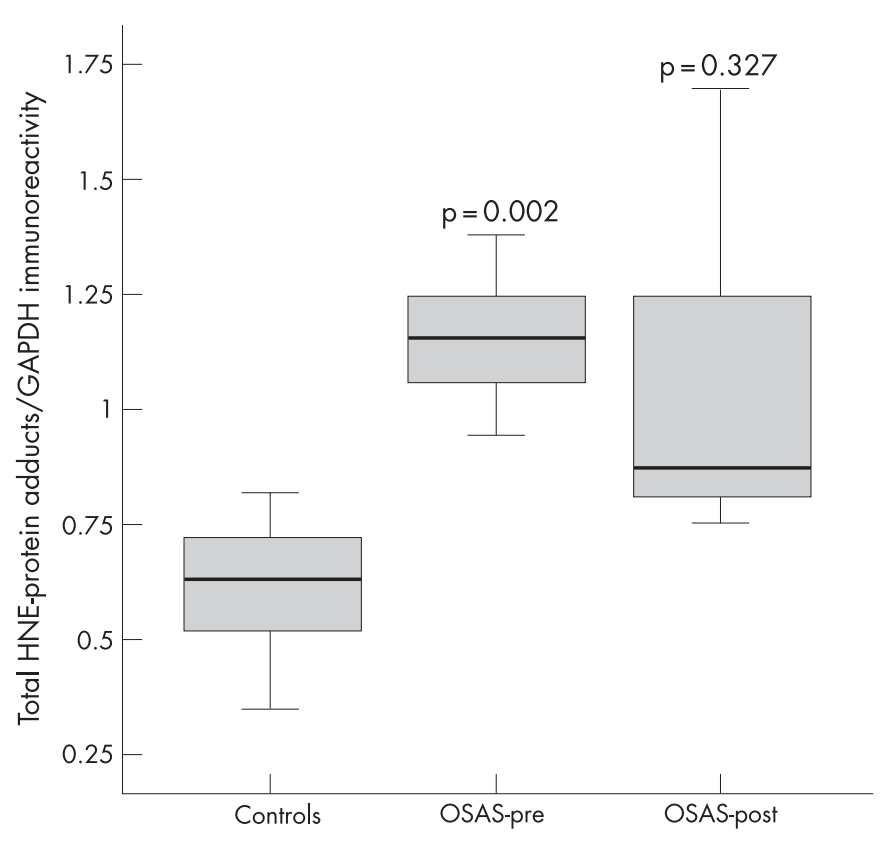

Figure 3 Optical densities in the box plots are expressed as the ratio of the optical densities of total hydroxynonenal (HNE)-protein adducts to those of glyceraldehyde-3-phosphate dehydrogenase (GAPDH). Standard box plots with median (25th and 75th percentiles) and whiskers (at minimum and maximum values) are shown. At baseline, total levels of HNE-protein adducts were significantly greater in the external intercostal muscles of patients with severe obstructive sleep apnoea syndrome (OSAS) than in control muscles (median values 1.15 and 0.63 , respectively, $p=0.002$ ). Total muscle HNE-protein adducts were not significantly modified after a 6month treatment period with continuous positive airway pressure (CPAP) (median value $0.87, p=0.327$ ). OSAS-pre, patients with OSAS before treatment with CPAP; OSAS-post, patients with OSAS after 6 months of treatment with CPAP.

OSAS and controls. No significant differences in the intensity of manganese superoxide dismutase (median values 0.99 and 0.88 , respectively, $\mathrm{p}=0.437$ ) or catalase (median values 1.13 and 1.09 , respectively, $\mathrm{p}=0.964$ ) were observed between the two groups. The levels of manganese superoxide dismutase (median value 0.66 ) or catalase (median value 1.23) were not significantly modified after treatment with CPAP in patients with OSAS $(p=0.674$ and $p=0.263$, respectively).

\section{DISCUSSION}

The main findings of this study are that, in the external intercostal muscles of patients with OSAS compared with those from control subjects:

- the endurance time of the respiratory muscles was significantly reduced;

- reactive carbonyl group levels were significantly increased and inversely correlated with the nadir $\mathrm{SaO}_{2}$ and had an almost significant inverse relationship with the endurance time;

- both MDA-protein and HNE-protein adducts were significantly greater and were correlated with the severity of the disease as measured by the AHI;

- protein tyrosine nitration was significantly increased and also correlated with the severity of the disease;

- the levels of the antioxidant enzymes were not significantly modified; and

- the endurance time of the respiratory muscles and the levels of these four indices of oxidative stress remained unmodified after a 6-month period of treatment with CPAP.

\section{Study model critique}

Open thoracotomy is the gold standard technique to obtain biopsy specimens from the diaphragm, the main inspiratory muscle. However, this surgical practice can only be used for subjects with serious illnesses such as lung neoplasms. Furthermore, the external intercostal muscles were also shown to be the primary inspiratory muscles during apnoea in a subpopulation of patients with OSAS. ${ }^{2}$ Finally, clear accessibility of the external intercostal muscles by means of an open biopsy on an outpatient basis at two different time points, as well as the fact that this model enabled us to carefully select the subjects excluding any co-morbid condition, clearly justified the design of the current study.

\section{Muscle structure and function}

Other reports in the literature have already shown the proportions of types I and II fibres in the quadriceps and genioglossus muscles of patients with OSAS. ${ }^{24}{ }^{25}$ The current study, however, is the first report on the morphometric analysis of one of the main inspiratory muscles, the external intercostal, in these patients. We have found a significant increase in the proportion of type I fibres in the intercostal muscles of patients with severe OSAS compared with controls. As these muscles must generate repeated bouts of increasing intrathoracic negative pressures in the face of apnoea throughout the entire night, one could argue that their fibres might switch to a more fatigue resistant phenotype, similar to that seen in other respiratory muscles in patients with chronic respiratory diseases such as COPD. ${ }^{12}$ However, respiratory muscle endurance time was significantly reduced in our population of patients with OSAS compared with control subjects of similar anthropometric characteristics. Although this could be somehow paradoxical, the fact that the respiratory muscles of patients with OSAS do not rest sufficiently during night time, and that their increased activity takes place during this part of the day, might account for these results.

\section{Muscle oxidative stress before CPAP treatment}

The present study is the first to provide evidence of the effects of ROS on muscle proteins and lipids of human intercostal muscles in severe OSAS. Oxidative stress, defined as the imbalance between oxidant production and antioxidant defences in favour of the former, has been implicated in the pathogenesis of several chronic conditions ${ }^{56}$ and in aging. ${ }^{7}$ It has also recently been suggested that oxidative stress is involved in the pathophysiology of OSAS, especially in that of cardiovascular morbidity. ${ }^{9}$

The inspiratory muscles of patients with OSAS do not rest sufficiently during night time because they have to generate increasing negative intrathoracic pressures to overcome upper airway obstruction. The lack of an adequate rest at night for the ventilatory muscles in OSAS might have deleterious consequences on muscle aerobic metabolism ${ }^{26}$ and on their daytime function, ${ }^{27}$ as well as on their antioxidant defences which, in turn, would increase oxidative stress. ${ }^{28}$ These negative biochemical events due to sleep deprivation probably counteract the training-like effects of the respiratory muscles secondary to the continuous ventilatory efforts. Furthermore, since our patients had very severe OSAS, as revealed by their AHI, it could be argued that the strong muscle contractions generated by their inspiratory muscles during the night might lead to a further increase in ROS production than that neutralised by their antioxidant systems. In fact, the patients with more severe OSAS were those with higher levels of oxidative and nitrosative stress in their intercostal muscles. Also, the higher percentage of oxidative fibres observed in the external intercostal muscles 

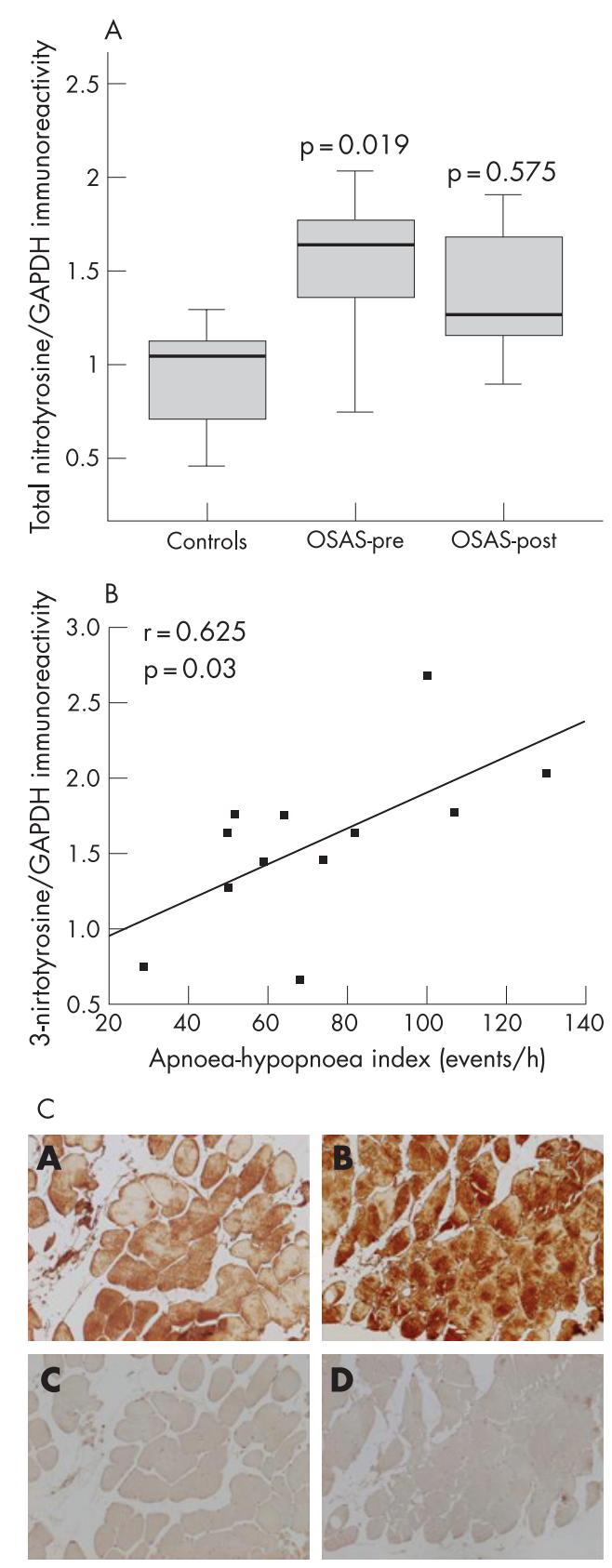

Figure 4 (A) Optical densities in the box plots are expressed as the ratio of the optical densities of total 3-nitrotyrosine immunoreactivity to those of glyceraldehyde-3-phosphate dehydrogenase (GAPDH). Standard box plots with median (25th and 75th percentiles) and whiskers (at minimum and maximum values) are illustrated. At baseline, total levels of protein tyrosine nitration were significantly greater in the external intercostal muscles of patients with severe obstructive sleep apnoea syndrome (OSAS) than in control muscles (median values 1.64 and 1.05, respectively, $p=0.019$ ). Total muscle protein tyrosine nitration was not significantly modified after a 6-month treatment period with continuous positive airway pressure (CPAP) (median value 1.27, $p=0.575$ ). OSAS-pre, patients with OSAS before treatment with CPAP; OSAS-post, patients with OSAS after 6 months of treatment with CPAP. (B) Overall, in patients with OSAS the ratio of the optical densities of total 3-nitrotyrosine immunoreactivity to those of GAPDH directly and significantly correlated with the apnoeahypopnoea index (events/h). (C) Immunohistochemical localisation of protein tyrosine nitration within the fibres of the external intercostal muscles of one control subject (panel A) and one patient with severe OSAS (panel B) (magnification 200 $\times$ ). Anti-3-nitrotyrosine antibody detected positive staining diffusely localised within the muscle fibres (panels A and B). Removal of primary anti-3-nitrotyrosine antibody completely eliminated positive protein tyrosine nitration staining (panels $C$ and D). Note that the staining was more intense in the intercostal muscles of the patient than in the control muscle. of our patients with OSAS before CPAP treatment would favour the increased production of oxidants within those fibres.

On the other hand, the recurrent upper airway obstruction during sleep leads to continuous hypoxia-reoxygenation cycles which, to a much lesser extent, resemble ischaemia-reperfusion effects on tissues. As this phenomenon was already shown to increase oxidative stress in patients with OSAS, ${ }^{89}$ it is reasonable to assume that continuous hypoxia-reoxygenation cycles occurring in the external intercostal muscles of our patients might also have contributed to enhance their oxidant production. Indeed, the patients with OSAS with the lowest nadir $\mathrm{SaO}_{2}$ values were those with higher levels of protein carbonylation in their external intercostal muscles. Moreover, our patients spent $80 \%$ of their sleep time at arterial oxygen saturations $<90 \%$, showing clear hypoxaemia during the night (mean $\mathrm{SaO}_{2} \approx 84 \%$, nadir $\mathrm{SaO}_{2} \approx 65 \%$ ), and chronic hypoxaemia has recently been shown to increase muscle oxidative stress in patients with COPD. $^{29}$

The muscle content of the two antioxidant systems analysed in the current study showed similar levels to those observed in the external intercostal muscles of the control individuals. In fact, the antioxidant enzyme paraxonase-1 was shown to be reduced in another population of OSAS patients with cardiovascular disease. ${ }^{9}$ As previously reported, ${ }^{30}$ one could also speculate from these findings that, in patients with OSAS, either the content or the activity of these enzymes might be altered as well as the regulatory mechanisms responsible for their upregulation, enhancing muscle oxidative stress.

\section{Muscle oxidative stress after CPAP treatment}

CPAP treatment has been proved to be beneficial in reducing both apnoea-hypopnoea events and oxygen desaturations, in inducing reparative sleep, and may improve long-term survival of patients with OSAS. ${ }^{31}$ In our study a 6-month period of treatment with CPAP significantly improved some lung function parameters, reduced the severity of the disease in all patients, and significantly improved oxygen desaturations (mean $\mathrm{SaO}_{2}$, nadir $\mathrm{SaO}_{2}$ and time spent below $90 \% \mathrm{SaO}_{2}$ ), and modified the proportions of muscle fibres to values observed in the control muscles. However, endurance time of the respiratory muscles, muscle protein carbonylation and nitration, and the antioxidant enzymes did not improve significantly after CPAP treatment. All our patients with OSAS had a high level of adherence to treatment with CPAP, with a mean usage time of $\geqslant 4 \mathrm{~h} /$ night. Moreover, the ESS score decreased significantly after CPAP treatment to normal values $(<10)$.

These findings can be explained by the fact that treatment with CPAP does not restore the optimal resting length of the inspiratory muscles. In fact, long-term treatment with CPAP increases lung volumes in patients with OSAS and normal lung function. ${ }^{32}$ In other words, despite the beneficial effects of CPAP treatment on physiological and clinical parameters, the inspiratory muscles remain in a disadvantageous state, hampering oxidative stress neutralisation. In this context, the loads imposed on the inspiratory muscles by the CPAP treatment would account for the persistence of increased levels of biomarkers of muscle oxidative stress and reduced endurance in our patients with OSAS.

The metabolic syndrome has also recently been associated with increased systemic oxidative stress levels. ${ }^{33}$ In our study the proportions of patients and controls with metabolic syndrome were similar (42\% and 50\%, respectively) and the increase in muscle oxidative stress biomarkers among the patients was independent of the metabolic syndrome. Moreover, after CPAP treatment muscle oxidative stress levels did not differ significantly between OSAS patients with metabolic syndrome and those without it (data not shown). 
We therefore conclude that OSAS, rather than the metabolic syndrome, contributes more to the increased oxidative stress levels in the external intercostal muscles of our patients.

In summary, molecular events occurring within the intercostal muscle fibres before CPAP treatment as a result of inadequate night time rest, chronic hypoxaemia, reduced muscle aerobic metabolism and the inefficiency of the antioxidant mechanisms might play a role in the respiratory muscle dysfunction observed in our population of patients with severe OSAS. CPAP treatment, however, failed to offset those deleterious effects on the intercostal muscles. It is also worth mentioning that, in a previous study by our group, ${ }^{4}$ it was shown that the reduced endurance of the respiratory muscles of patients with less severe OSAS was significantly improved after 2 months of treatment with CPAP. The fact that the patients in the present study had much more severe OSAS than those recruited in the former study might account for this discrepancy.

\section{CONCLUSIONS}

Our results provide first evidence that, in severe OSAS, the external intercostal muscles exhibit increased levels of oxidative stress and such levels are associated with the severity of the disease. A high level of adherence to CPAP treatment for 6 months does not significantly decrease these levels or improve respiratory muscle endurance, suggesting that inspiratory muscle performance is not completely restored after CPAP.

\section{ACKNOWLEDGEMENTS}

The authors thank Francesc Sánchez, Sandra Mas and Beatriz de la Puente for their technical assistance in the laboratory and Roger Marshall for his assistance with editing.

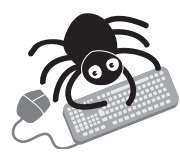

Further information is given in the online supplement available at http://thorax.bmj.com/supplemental.

\section{Authors' affiliations}

Esther Barreiro, Joaquim Gea, Muscle and Respiratory System Research Unit and Respiratory Medicine Department, IMIM-Hospital del Mar, Centro de Investigación en Red de Enfermedades Respiratorias (CibeRes),

Experimental Sciences and Health Department (CEXS), Universitat Pompeu Fabra, PRBB, Barcelona, Catalonia, Spain

Adam Nowinski, Pawel Sliwinski, Department of Respiratory Medicine, Institute of Tuberculosis and Lung Diseases, Warsaw, Poland

This study was supported by the European Network for the study of clinical and biological implications of Respiratory Muscle failure in patients with Chronic Obstructive Pulmonary Disease (ERESMUS in COPD) (BMTH4CT98-3406) (EU) and RESPIRA (RTIC C03/11) (Spain).

Competing interests: None.

\section{REFERENCES}

1 Young T, Palta M, Dempsey J, et al. The occurrence of sleep-disordered breathing among middle-aged adults. N Engl J Med 1993;328:1230-5.

2 Wilcox PG, Pare PD, Road JD, et al. Respiratory muscle function during obstructive sleep apnea. Am Rev Respir Dis 1990;142:533-9.

3 Shivaram U, Cash ME, Beal A. Nasal continuous positive airway pressure in decompensated hypercapnic respiratory failure as a complication of sleep apnea. Chest 1993;104:770-4.

4 Aran X, Felez MA, Gea J, et al. Respiratory muscle force and resistance in patients with SAHS. The effect of using nighttime CPAP. Arch Bronconeumol $1999 \cdot 35: 440-5$.
5 Wu BJ, Kathir K, Witting PK, et al. Antioxidants protect from atherosclerosis by a heme oxygenase-1 pathway that is independent of free radical scavenging. J Exp Med 2006;203:1117-27.

6 Butterfield DA, Poon HF, St Clair D, et al. Redox proteomics identification of oxidatively modified hippocampal proteins in mild cognitive impairment: Insights into the development of Alzheimer's disease. Neurobiol Dis 2006;22:223-32.

7 Barreiro E, Coronell C, Lavina B, et al. Aging, sex differences, and oxidative stress in human respiratory and limb muscles. Free Radic Biol Med 2006:41:797-809.

8 Schulz R, Mahmoudi S, Hattar K, et al. Enhanced release of superoxide from polymorphonuclear neutrophils in obstructive sleep apnea. Impact of continuous positive airway pressure therapy. Am J Respir Crit Care Med 2000;162:566-70.

9 Lavie L, Vishnevsky A, Lavie P. Evidence for lipid peroxidation in obstructive sleep apnea. Sleep 2004;27:123-8.

10 Barreiro E, Gea J, Corominas JM, et al. Nitric oxide synthases and protein oxidation in the quadriceps femoris of COPD patients. Am J Respir Cell Mol Biol 2003;29:771-8

11 Koechlin C, Couillard A, Simar D, et al. Does oxidative stress alter quadriceps endurance in chronic obstructive pulmonary disease? Am J Respir Crit Care Med 2004:169:1022-7.

12 Barreiro E, de la Puente B, Minguella, et al. Oxidative stress and respiratory muscle dysfunction in severe chronic obstructive pulmonary disease. Am J Respir Crit Care Med 2005;171:1116-24.

13 Barreiro E, Gea J, Matar G, et al. Expression and carbonylation of creatine kinase in the quadriceps femoris muscles of patients with chronic obstructive pulmonary disease. Am J Respir Cell Mol Biol 2005;33:636-42.

14 Barreiro E, Sanchez D, Galdiz JB, et al. N-acetylcysteine increases manganese superoxide dismutase activity in septic rat diaphragms. Eur Respir $J$ 2005;26:1032-9.

15 Glenmark B. Skeletal muscle fibre types, physical performance, physical activity and attitude to physical activity in women and men. A follow-up from age 16 to 27. Acta Physiol Scand Suppl 1994;623:1-47.

16 Quanjer PH, Tammeling GJ, Cotes JE, et al. Symbols, abbreviations and units. Working Party Standardization of Lung Function Tests, European Community for Steel and Coal. Eur Respir J Suppl 1993;16:85-100.

17 Black LF, Hyatt RE. Maximal respiratory pressures: normal values and relationship to age and sex. Am Rev Respir Dis 1969:99:696-702.

18 ATS Committee on Proficiency Standards for Clinical Pulmonary Function Laboratories. ATS statement: guidelines for the six-minute walk test. Am J Respir Crit Care Med 2002;166:111-7.

19 Enright PL, Sherrill DL. Reference equations for the six-minute walk in healthy adults. Am J Respir Crit Care Med 1998;158:1384-7.

20 Mathiowetz V, Kashman N, Volland G, et al. Grip and pinch strength: normative data for adult. Arch Phys Med Rehabil 1985;66:69-74.

21 American Academy of Sleep Medicine Task Force. Sleep related breathing disorders in adults: recommendation for syndrome definition and measurement techniques in clinical research. Sleep 1999:22:667-89.

22 Johns MW. A new method for measuring daytime sleepiness: the Epworth sleepiness scale. Sleep 1991;14:540-5.

23 Ramirez-Sarmiento A, Orozco-Levi M, Guell R, et al. Inspiratory muscle training in patients with chronic obstructive pulmonary disease: structural adaptation and physiologic outcomes. Am J Respir Crit Care Med 2002;166:1491-7.

24 Carrera M, Barbe F, Sauleda J, et al. Patients with obstructive sleep apnea exhibit genioglossus dysfunction that is normalized after treatment with continuous positive airway pressure. Am J Respir Crit Care Med 1999;159:1960-6.

25 Sauleda J, Garcia-Palmer FJ, Tarraga S, et al. Skeletal muscle changes in patients with obstructive sleep apnoea syndrome. Respir Med 2003:97:804-10.

26 Vondra K, Brodan V, Bass A, et al. Effects of sleep deprivation on the activity of selected metabolic enzymes in skeletal muscle. Eur J Appl Physiol Occup Physiol 1981;47:41-6.

27 Cooper KR, Phillips BA. Effect of short-term sleep loss on breathing. J Appl Physiol 1982;53:855-8.

28 Everson CA, Laatsch CD, Hogg N. Antioxidant defense responses to sleep loss and sleep recovery. Am J Physiol Regul Integr Comp Physiol 2005;288:R374-83.

29 Koechlin C, Maltais F, Saey D, et al. Hypoxaemia enhances peripheral muscle oxidative stress in chronic obstructive pulmonary disease. Thorax 2005:60:834-41.

30 MacMillan-Crow LA, Crow JP, Thompson JA. Peroxynitrite-mediated inactivation of manganese superoxide dismutase involves nitration and oxidation of critical tyrosine residues. Biochemistry 1998;37:1613-22.

31 Marin JM, Carrizo SJ, Vicente E, et al. Long-term cardiovascular outcomes in men with obstructive sleep apnoea-hypopnoea with or without treatment with continuous positive airway pressure: an observational study. Lancet 2005;365: 1046-53.

32 Verbraecken J, Willemen M, De Cock W, et al. Continuous positive airway pressure and lung inflation in sleep apnea patients. Respiration 2001;68:357-64

33 Van Guilder GP, Hoetzer GL, Greiner JJ, et al. Influence of metabolic syndrome on biomarkers of oxidative stress and inflammation in obese adults. Obesity 2006;14:2127-31. 